\title{
Emissivity measurements of reflective surfaces at near-millimeter wavelengths
}

\author{
J. J. Bock, M. K. Parikh, M. L. Fischer, and A. E. Lange
}

\begin{abstract}
We have developed an instrument for directly measuring the emissivity of reflective surfaces at near-millimeter wavelengths. The thermal emission of a test sample is compared with that of a reference surface, allowing the emissivity of the sample to be determined without heating. The emissivity of the reference surface is determined by one's heating the reference surface and measuring the increase in emission. The instrument has an absolute accuracy of $\Delta \epsilon=5 \times 10^{-4}$ and can reproducibly measure a difference in emissivity as small as $\Delta \epsilon=10^{-4}$ between flat reflective samples. We have used the instrument to measure the emissivity of metal films evaporated on glass and carbon fiber-reinforced plastic composite surfaces. We measure an emissivity of $(2.15 \pm 0.4) \times 10^{-3}$ for gold evaporated on glass and $(2.65 \pm 0.5) \times 10^{-3}$ for aluminum evaporated on carbon fiber-reinforced plastic composite.

Key words: Emissivity, reflectance, millimeter, infrared, mirrors.
\end{abstract}

\section{Introduction}

The sensitivity of IR and millimeter-wave telescopes operated at ambient temperature can be severely limited by thermal emission from the telescope itself, especially for balloonborne and orbital telescopes for which atmospheric emission is negligible. Even for off-axis systems designed to minimize the emissivity of the telescope, emission from warm mirror surfaces may dominate the photon background viewed by the detector.1,2 The thermal emission from a simple $300 \mathrm{~K}$ mirror surface with an emissivity $\epsilon=2.5 \times$ $10^{-3}$ is, for example, 10 times brighter than the astrophysical sky at $\lambda=1 \mathrm{~mm}$.

Telescope emission can limit sensitivity in two ways. At a minimum, quantum fluctuations in the total power reaching the detector set the fundamental sensitivity limit. In addition, telescope emission can also produce noise and systematic errors in photometry from drifts in the telescope temperature and nonuniformity of the emission across the mirror sur-

J. J. Bock and A. E. Lange are with the Division of Physics, Mathematics and Astronomy, Mail Code 424-47, California Institute of Technology, Pasadena, California 91125. M. K. Parikh is with the Department of Physics, Princeton University, Princeton, New J ersey 08544. M. L. Fischer is with the Indoor Environment Program, Building 90-3058, Lawrence Berkel ey Laboratory, 1 Cycletron Road, Berkeley, California 94720.

Received 31 October 1994; revised manuscript received $24 \mathrm{~J}$ anuary 1995.

0003-6935/95/224812-05\$06.00/0.

(c) 1995 Optical Society of America. faces. For these reasons, it is important to minimize the emissivity of mirror surfaces.

The millimeter-wave emissivity of a bulk metal surface can be as low as several tenths of a percent. Bulk metal mirrors are, however, prohibitively massive for many applications. Recent advances in composite mirror technology permit large, lightweight mirrors to be fabricated that have diffraction-limited image quality at infrared and millimeter wavelengths. ${ }^{3,4}$ The emissivity of composite mirrors is a function of the thickness and quality of the reflective coating and of the microscopic roughness of the composite surface, however, and must thus be carefully characterized to ensure that it approaches that of bulk metal.

We have developed an instrument to determine the emissivity of room-temperature reflective surfaces at near-millimeter wavelengths. The instrument is capable of measuring the emissivity of a surface with an absolute accuracy of $\Delta \epsilon=5 \times 10^{-4}$ and can reproducibly measure differences in emissivity of $\Delta \epsilon=1 \times$ $10^{-4}$. The measurements can be made without one's heating the sample, allowing the emissivity of thermally insulating materials such as carbon fiberreinforced plastic (CFRP) composite mirrors to be accurately measured.

\section{Emissivity of Metal Films}

The emissivity of a metal film deposited on a dielectric surface depends on the thickness and the surface electrical conductivity of the film. Incident radiation may be absorbed in the metal film, transmitted 
through the film, or reflected. We assume that any radiation transmitted through the film is absorbed by material that is at the same temperature as the film. In this limit the effective emissivity of a metal-coated dielectric surface is given by $\epsilon \equiv 1-R$, where $R$ is the reflectivity.

The emissivity of bulk metal at normal incidence is given by the Hagen-Rubens formula ${ }^{5}$

$$
\epsilon=\left(\frac{16 \pi \mathrm{C} \epsilon_{0}}{\lambda \sigma}\right)^{1 / 2}(\mathrm{mks}),
$$

where $\lambda$ is the wavelength, $\epsilon_{0}$ is the permittivity of free space, $c$ is the speed of light, and $\sigma$ is the surface el ectrical conductivity. For a metal film the emissivity approaches the bulk behavior if the thickness of the film is $\geq 2$ times larger than the skin depth $\delta$ over which the el ectric field is attenuated by a factor of $e$,

$$
\delta=\left(\frac{\lambda}{\pi \mathrm{C} \sigma \mu}\right)^{1 / 2}(\mathrm{mks}),
$$

where $\mu$ is the magnetic permeability of the film.

The calculated emissivity of an aluminum-coated dielectric, plotted in Fig. 1, approaches that of bulk aluminum for a metal film thickness, $\mathrm{t} \geqslant 2 \delta=300$ $(\lambda / \mathrm{mm})^{1 / 2} \mathrm{~nm}$. For example, a 1- $\mu$ m coating of al uminum is sufficiently thick to provide a surface with minimal emissivity for $\lambda<1 \mathrm{~cm}$.

The bulk limit is thus a useful approximation of the emissivity. However, it may be difficult for practical reasons to coat large surfaces with more than several hundred nanometers of metal because of adhesion problems. Moreover the risk of separation of a metal film from the dielectric backing because of differential thermal contraction increases with film thickness. Hence it is desirable to use the minimum

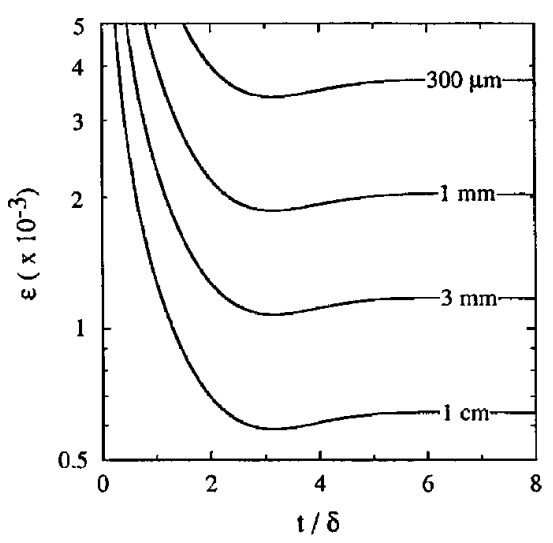

Fig. 1. Calculated effective emissivity $(\epsilon=1-R)$ of aluminumcoated composite mirrors plotted as a function of film thickness over skin depth $\mathrm{t} / \delta$ for wavel engths $\lambda=1 \mathrm{~cm}, 3 \mathrm{~mm}, 1 \mathrm{~mm}$, and 300 $\mu \mathrm{m}$. We calculate the emissivity for an incident angle of $45^{\circ}$, averaging over polarization, assuming the electrical conductivity of bulk aluminum $\sigma=3.7 \times 10^{7} \Omega^{-1} \mathrm{~m}^{-1}$. Theskin depth depends on the electrical conductivity and the wavelength [see Eq. (2)]. For bulk aluminum, $\delta=150(\lambda / \mathrm{mm})^{1 / 2} \mathrm{~mm}$. The emissivity can be extrapolated with high accuracy to other wavel engths and electrical conductivities by scaling the curves by $\epsilon^{\prime}=\epsilon\left(\lambda \sigma / \lambda^{\prime} \sigma^{\prime}\right)^{1 / 2}$. film thickness that gives acceptable performance. Because the actual electrical conductivity of a thin film depends on purity and surface effects, a precise measurement of the emissivity is necessary.

\section{Instrument}

The instrument is designed to measure the difference in emissivity between reflective surfaces by comparing the thermal emission of a test sample with that of a reference surface. One determines the absolute emissivity of the reference surface by heating the reference surface and measuring the increase in emission. The instrument, shown schematically in Fig. 2, consists of a detector, an ellipsoidal mirror, a sample holder placed behind a reflective chopper and screen, and a liquid-nitrogen load.

The detector is a superfluid ${ }^{4} \mathrm{He}$ composite bolometer ${ }^{6}$ placed behind a cooled filter stack. The bolometer has an el ectrical noise-equivalent power (NEP) of $3 \times 10^{-15} \mathrm{~W} \mathrm{~Hz}^{-1 / 2}$ and a responsivity of $1.2 \times 10^{6}$ $\mathrm{V} / \mathrm{W}$ and remains linear to within $2 \%$ over the range of optical loading present in the experiment. The cooled filter stack, consisting of black polyethylene, ${ }^{7}$ Fluorogold ${ }^{8}$ a glass-bead filter ${ }^{9}{ }^{9}$ metal-mesh filter, ${ }^{10}$ and a thick grill filter, ${ }^{11}$ defines the passband and provides a high degree of rejection of short-wavelength radiation. Thespectral response of theinstrument, shown in Fig. 3, gives a $\Delta \lambda / \lambda=0.29$ bandpass centered at a wavelength of $1.33 \mathrm{~mm}$. For a Rayleigh-J eans spectrum the weighted band center is $\lambda=$ $1.16 \mathrm{~mm}$. For a surface with an emissivity $\epsilon \propto \lambda^{-1 / 2}$, the emissivity is determined at $\lambda=1.17 \mathrm{~mm}$. The short-wavelength response is measured to be $<2 \times$ $10^{-3}$ of the peak response for $750 \mu \mathrm{m}>\lambda>275 \mu \mathrm{m}$. The waveguide cutoff of the thick grill filter provides

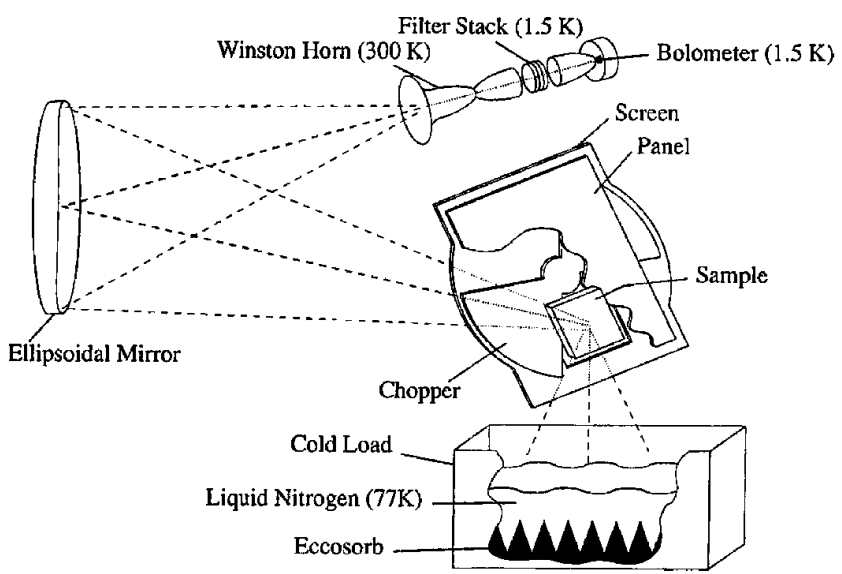

Fig. 2. Schematic of the emissivitometer. In the time-reversed sense, radiation detected by the bolometer passes through a cold filter stack and is collimated by a room-temperature back-to-back Winston horn with an apodizing flare. The radiation reflects off an off-axis ellipsoidal mirror, passes through a screen, is modulated by a chopper, passes through a panel, and is focused onto a reflective sample mounted on the panel. The radiation is reflected into a $77-\mathrm{K}$ cold load alternately by the reflective chopper blade and by the sample. The cold load consists of foam E ccosorb submerged in a bath of liquid nitrogen. 


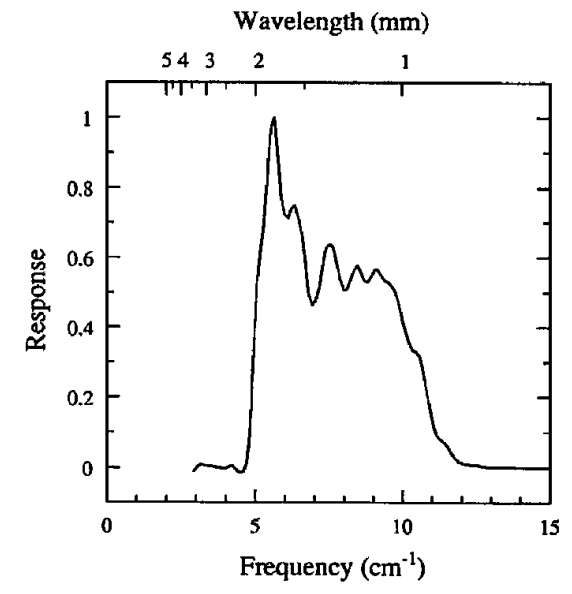

Fig. 3. Spectral response of the instrument.

excellent rejection ${ }^{11}$ of long-wavelength radiation for $\lambda>2.2 \mathrm{~mm}$.

A room-temperature Winston feedhorn ${ }^{12}$ with an apodizing flare to reduce diffraction couples the radiation to the detector and defines the detector field of view. The Winston feedhorn is positioned at one focus of the off-axis ellipsoidal mirror. The sample to be measured is placed at the other focus, behind a 4.5 $\mathrm{cm} \times 4.5 \mathrm{~cm}$ aperture in an aluminum panel. A reflective chopper blade mounted on the panel modulates the detector's view of the sample at $5 \mathrm{~Hz}$. The sample, panel, and chopper are positioned so that the detector views a 77-K cold load alternately in reflection from the sample and the chopper blade. The cold load consists of 5-cm-thick, cone-shaped foam Eccosorb,13 a millimeter-wave absorbing material, which is submerged in a bath of liquid nitrogen.

Several precautions are taken to reduce spurious signals from stray radiation. The Winston feedhorn has an apodizing flare to reduce diffraction of radiation into the detector. The edges of the ellipsoidal mirror are baffled with Eccosorb foam to intercept radiation spilling past the mirror edge. Finally, an aluminum screen is placed in front of the panel and chopper assembly to minimize modulation of stray radiation by the chopper.

\section{Method}

The output of the detector is electronically amplified and demodulated by a lock-in amplifier referenced to the drive frequency of the chopper. The instrument is calibrated by removal of the reflective sample from the aluminum panel so that the detector alternately views the room and the cold load in reflection off the chopper. We define the brightness of a blackbody source in Rayleigh-J eans antenna-temperature units, so that its brightness is given by the temperature of the source. The responsivity $\mathrm{G}$ of the instrument is given by

$$
\mathrm{G}=\mathrm{S}_{\mathrm{cal}} /\left(\text { Troom }-T_{\mathrm{road}}\right)(\mathrm{V} / \mathrm{K}) \text {, }
$$

where $S_{\text {cal }}$ is the calibration signal and room and Toad are the antenna temperatures of the room and the cold load, respectively. We assume that the cold load is $(95 \pm 5) \%$ absorbing, giving room - Toad = $(207 \pm 11) \mathrm{K}$. We use the calibration signal to define the phase of the optical signal with respect to the reference drive signal from the chopper.

When a reflective sample is placed in the aperture in the aluminum panel, the signal from the detector is given by

$$
\mathrm{S}=\mathrm{G}\left[\epsilon_{\mathrm{s}}\left(T_{\mathrm{s}}-\text { Toad }_{\text {oad }}-\epsilon_{\mathrm{ch}}\left(\mathrm{T}_{\mathrm{ch}}-\text { Toad }_{\text {oad }}\right)\right]+\mathrm{S}_{\text {offset }}(\mathbf{V}),\right.
$$

where $\epsilon_{\mathrm{s}}$ and $\mathrm{T}_{\mathrm{s}}$ are the emissivity and the thermal temperature of the sample and $\epsilon_{\mathrm{ch}}$ and $\mathrm{T}_{\mathrm{ch}}$ are the emissivity and the thermal temperature of the chopper blade. The term $\mathrm{S}_{\text {offset }}$ is due to an instrumental offset discussed in Section 5. Assuming that $\mathrm{S}_{\text {offset }}$ remains stable, we can determine the difference in emissivity between a sample and a reference surface, $\Delta \epsilon_{\mathrm{s}}=\epsilon_{\mathrm{s}}-\epsilon_{\mathrm{ref}}$, by differencing the signals obtained in subsequent measurements of the sample and the reference surface,

$$
\Delta \epsilon_{\mathrm{s}}=\mathrm{G}^{-1}\left(\frac{\mathrm{S}_{\mathrm{s}}-\mathrm{S}_{\text {ref }}}{\text { Troom }- \text { Toad }}\right)=\frac{\Delta \mathrm{S}}{\mathrm{S}_{\mathrm{cal}}},
$$

where $\Delta \mathrm{S}=\mathrm{S}_{\mathrm{s}}-\mathrm{S}_{\text {ref }}$ and we have assumed $\mathrm{T}_{\mathrm{S}}=\mathrm{T}_{\text {ref }}$ room. Note that the determination of the difference emissivity is independent of the determination of the responsivity $\mathrm{G}$.

The absolute emissivity of a reference sample is obtained by placing it into the detector beam and heating the reference sample. The change in the signal $\Delta \mathrm{S}$ caused by a change in the reference sample temperature $\Delta T_{s}$ [see Eq. (4)] can be determined in the presence of a stable instrumental offset and gives the emissivity of the reference surface,

$$
\epsilon_{\mathrm{s}}=\Delta \mathrm{S} /\left(\mathrm{G} \Delta \mathrm{T}_{\mathrm{s}}\right) .
$$

The reference sample must have a uniform temperature and therefore must be thermally conductive.

Scattering at the sample surface will increase the measured emissivity when the differencing technique is used, because radiation scattered through large angles originates at ambient temperature rather than in the cold load. Thus the quantity that is actually measured by this method is the sum of the emission and the large-angle scattering. This is an upper limit on the emissivity and is the quantity of interest in estimating the effective emissivity of a telescope in which radiation scattered through large angles by the mirror surface is eventually intercepted by a warm surface. Scattering at the surface of the reference sample does not affect the emissivity determined by heating the sample.

\section{Measurements}

Careful alignment of the instrument is necessary before measurements are carried out. We place a small optical lamp at the position of the Winston horn and adjust the position of the ellipsoidal mirror and 
the sample to obtain the best image of the lamp on the sample. The angle of the aluminum panel and the position of the cold load are then adjusted so that all the radiation from the optical lamp is reflected into the cold load by both the sample and the chopper blade. Wethen remove the lamp and carefully insert the detector system so that the position of the Winston horn coincides with the position of the lamp.

When an aluminum reference surface is placed on the aluminum panel, we measure an instrumental offset of $\epsilon_{\text {offset }}=\mathrm{G}^{-1} \mathrm{~S}_{\text {offset }}(\text { room }- \text { Toad })^{-1} \sim 5 \times 10^{-3}$, which is $\sim 80^{\circ}$ out of phase from the optical signal. An out-of-phase offset may be produced by radiation scattering off the edges of the chopper blade onto highly emissive room-temperature surfaces. An offset may also be produced by a misalignment between the chopper blade and the sample. Because the instrument compares the emission between samples, it is susceptible to the instrumental offset only if the offset is unstable. We monitor the offset signal over $4 \mathrm{~h}$ and find it to be extremely stable, with a drift of $\mathrm{d} \epsilon_{\text {offset }} / \mathrm{dt}=4 \times 10^{-5} / \mathrm{h}$ for the inphase signal and a drift of $\mathrm{d} \epsilon_{\text {offset }} / \mathrm{dt}<1.4 \times 10^{-5} / \mathrm{h}$ for the out-of-phase signal. Repeatedly removing and inserting a flat sample does not change the offset to within $\Delta \epsilon_{\text {offset }}=5$ $\times 10^{-5}$ for either phase.

We place a transmissive Styrofoam lid over the cold load during the differencing measurements to reduce any drifts associated with cooling of the baffles, chopper, or sample and to prolong the lifetime of the liquid nitrogen. We frequently monitor the instrumental offset by inserting the aluminum reference surface and occasionally monitor the calibration by removing the sample.

We measure the absolute emissivity of the reference surface by placing it in the detector beam and varying its temperature [see Eq. (6)]. For this measurement the col d load is not necessary and is removed. In the absence of the cold load the system offset becomes smaller, and we measure an offset of $\mathrm{G}^{-1} \mathrm{~S}_{\text {offset }}\left(\right.$ room - Toad $^{-1} \sim 1.4 \times 10^{-4}$ for the inphase signal and $\sim 3 \times 10^{-4}$ for the out-of-phase signal. The reference sample is suspended by a fibergl ass rod to isolate it thermally from the aluminum panel. Two thermometers are attached on the back surface of the reference sample to verify that any temperature gradients across the sample are small. The signal from the detector system is first recorded with the sample at room temperature. The sample is then heated to $\sim 70{ }^{\circ} \mathrm{C}$, and the signal from the detector system is recorded at several reference sample temperatures. Heating the baffles or the chopper can vary the instrumental offset during the course of the measurement. We monitor the instrumental offset both before and after heating the reference surface by placing a separate room-temperature alumi num surface in the beam. We thus determine that the uncertainty from a drifting instrumental offset gives a typical error of $\Delta \epsilon \sim 3 \times 10^{-4}$.

The reference surface is a 7-mm-thick polished block of 6061-T6 aluminum alloy. We also measure the emissivity of a brass reference surface and a reference surface consisting of a polished block of 6061-T6 aluminum al loy coated with $500 \mathrm{~nm}$ of evaporated bismuth. Thethickness of the bismuth coating is less than a skin depth so the emissivity of the sample is lower than that expected for bulk bismuth. The higher emissivities of the brass and the bismuthcoated reference surfaces are useful in the verification of the performance of the instrument.

\section{Results}

The results of the heating measurements shown in Table 1 include statistical error, the error from the stability of the offset, and a $5 \%$ overall calibration error. The sensitivity of the instrument is sufficient to detect an emissivity difference of $\Delta \epsilon=2 \times 10^{-4}$ in $1 \mathrm{~s}$ of integration. The accuracy with which absolute emissivity can be determined is limited by the uncertainty in the emissivity of the reference surface.

Wemeasuretheemissivity of several mirror samples with the differencing technique by comparing the emissivity of each sample with that of the aluminum reference surface and by calculating the difference in the emissivity between the samples with Eq. (5). When the mirror surfaces CFRP are tested, both the inphase and out-of-phase signals depend on the orientation and position of the sample. We think that the most likely cause of this variation is the result of the CFRP mirror samples being formed from a paraboloidal mold; thus they are not flat. Curved samples can vary the system offset by reflecting radiation into a different portion of the cold load than the reference surface. Variation in the emissivity over the sample cannot easily account for the observed variation in the signal when the composite samples are rotated. A rotational dependence in the emissivity, for example, caused by the orientation of the fibers or machining grooves on the composite surface, cannot explain the observed variation in the signal when different portions of the same sample are measured in a fixed orientation or when the sample is rotated by $180^{\circ}$. Only those composite samples that gave the smallest signal variation arelisted in Table 1 . These

Table 1. Measured Emissivities of Various Samples

\begin{tabular}{|c|c|c|}
\hline Sample & $\begin{array}{l}\text { Absolute } \\
\text { Emissivity } \\
\epsilon_{\mathrm{s}}\left(\times 10^{-3}\right)\end{array}$ & $\begin{array}{l}\text { Difference Emissivity } \\
\begin{array}{c}\epsilon_{\mathrm{s}}-\epsilon_{\mathrm{ref}}(\mathrm{Al} \text { ref }) \\
\left(\times 10^{-3}\right)\end{array}\end{array}$ \\
\hline Aluminum reference & $3.1 \pm 0.4^{b}$ & - \\
\hline Brass reference & $3.45 \pm 0.45^{b}$ & $0.55 \pm 0.1$ \\
\hline $\begin{array}{l}\text { Bismuth on aluminum } \\
\text { reference }\end{array}$ & $5.55 \pm 0.7^{b}$ & $2.1 \pm 0.1$ \\
\hline Aluminum on glass $(\mathbf{1})$ & $2.6 \pm 0.4$ & $-0.5 \pm 0.1$ \\
\hline Aluminum on glass (2) & $2.25 \pm 0.4$ & $-0.85 \pm 0.1$ \\
\hline Gold on glass & $2.15 \pm 0.4$ & $-0.95 \pm 0.1$ \\
\hline Aluminum on CFRP $(\mathbf{1})$ & $2.65 \pm 0.5$ & $-0.4 \pm 0.3$ \\
\hline Aluminum on CFRP (2) & $6.15 \pm 0.5$ & $3.1 \pm 0.3$ \\
\hline Aluminum on CFRP $(3)$ & $2.85 \pm 0.5$ & $-0.2 \pm 0.3$ \\
\hline
\end{tabular}

aE missivity determined at $\lambda=1.17 \mathrm{~mm}$ (see text).

bAbsolute emissivity determined with the heating technique. 
CFRP surfaces have a radius of curvature of $3 \mathrm{~m}$ and give a signal variation of typically $\Delta \epsilon=3 \times 10^{-4}$ with sample orientation. The signal variation for the reference samples and the evaporated glass samples was typically $\Delta \epsilon=1 \times 10^{-4}$. We calculated the emissivity of each sample tabulated in Table 1 by averaging measurements of the emissivity obtained for several different sample orientations.

The measured emissivity of the best aluminum surface, $(2.25 \pm 0.4) \times 10^{-3}$ for aluminum evaporated on glass, is nearly consistent with the calculated emissivity of bulk aluminum, which was $\epsilon=1.85 \times$ $10^{-3}$ at $\lambda=1.17 \mathrm{~mm}$ and $\theta=45^{\circ}$. The measured emissivity of the polished aluminum reference surface, $(3.1 \pm 0.4) \times 10^{-3}$, is somewhat higher than that of the evaporated surfaces possibly because of surface damage from polishing. The emissivity of the best aluminum-coated CFRP surface, $(2.65 \pm 0.5) \times 10^{-3}$, is similar to that of the aluminum-coated glass surfaces. The lowest measured emissivity is $(2.15 \pm 0.4) \times 10^{-3}$ for gold evaporated on glass. The emissivity of the bismuth-coated reference sample and the brass reference sample obtained with the differencing technique agree well with the emissivity obtained directly with the heating technique, confirming the performance of the instrument.

\section{Conclusions}

We have developed an instrument which relies on a simple differencing technique and does not involve heating the sample, to measure directly the emissivity of reflective surfaces at near-millimeter wavelengths. The measured emissivities of the best evaporated coatings are found to be comparable with those calculated for bulk metal surfaces. One may use the technique to measure the emissivity of any reflective surface or to determine the absorption of transmissive materials by placing the material in front of a reflecting surface. By employing a filter wheel or by replacing the cold load with a spectrometer, one can obtain spectral information.

The authors thank Sylvia Li for work with the instrument and acknowledge helpful conversations with Dave Cottingham and Paul Richards. This research was supported in part by the NASA graduate student research program fellowship NGT-50771.

\section{References and Notes}

1. M. L. Fischer, D. C. Alsop, E. S. Cheng, A. C. Clapp, D. A. Cottingham, J . O. Gundersen, T. C. Koch, E. Kreysa, P. R. Meinhold, A. E. Lange, P. M. Lubin, P. L. Richards, and G. F. Smoot, "A bolometric millimeter-wave system for observations of anisotropy in the cosmic microwave background radiation on medium angular scales," Astrophys. J . 388, 242-252 (1992).

2. A. E. Lange, J. J. Bock, and P. Mason, "The far-infrared explorer (FIRE)," in Infrared and Submilliter Space Missions in the Coming Decade, H. Thomson, M. Sauvage, P. Gallais, and L. Vigroux, eds., Space Sci. Rev. 74, 151-156 (1995).

3. S. S. Tompkins, D. E. Bowles, J. G. Funk, J . A. Lavoie, and T. W. Towell, "The development of composite materials for spacecraft precision reflector panels," in Advances in Optical Structure Systems, J. Breakwell, V. L. Genberg, and G. C. Krumweide, eds., Proc. Soc. Photo-Opt. Instrum. Eng. 1303, 512-523 (1990).

4. W. F. Hoffmann, "Light-weight composite mirrors for space submillimeter astronomy," in Space-Borne Sub-Millimeter Astronomy Mission, ESA SP 260, N. Longdon, ed. (European Space Agency Publications Division, Noordwijk, The Netherlands, 1986), pp. 231-238.

5. M. A. Born and E. Wolf, Principles of Optics (Pergamon, Oxford, 1975), pp. 611-633.

6. A. E. Lange, E. Kreysa, S. E. McBride, P. L. Richards, and E. E. Haller, "Improved fabrication techniques for infrared bolometers," I nt. J . I nfrared Millimeter Waves 4, 689-706 (1983).

7. K. D. Möller, D. J . McMahom, and D. R. Smith, "Far-infrared transmission of filters for the $300-18-\mathrm{cm}^{-1}$ spectral region," Appl. Opt. 5, 403-406 (1966).

8. M. Halpern, H. P. Gush, E. Wishnow, and V. De Cosmo, "Far infrared transmission of dielectrics at cryogenic and room temperatures: glass, Fluorogold, Eccosorb, Stycast, and various plastics," Appl. Opt. 25, 565-570 (1986).

9. S. Sato, S. Hayakawa, T. Matsumoto, H. Matsuo, H. Murakami, K. Sakai, A. E. Lange, and P. L. Richards, "Submillimeter wave low pass filters made of glass beads," Appl. Opt. 28, 4478-4481 (1989).

10. K. J . Leipoldt, T. Happich, E. Kreysa, and H. -P. Gemünd, "Scattering matrix methods for far-infrared metal mesh filters," I nt. J . I nfrared Millimeter Waves 12, 263-274 (1991).

11. T. Timusk and P. L. Richards, "Near millimeter wave bandpass filters," Appl. Opt. 20, 1355-1360 (1981).

12. R. Winston, "Light collection within the framework of geometrical optics,"J . Opt. Soc. Am. 60, 245-247 (1970).

13. Emerson \& Cuming, Inc., AN-72, Woburn, Mass. 01888. 